\title{
Resiliencia en la escuela: Un camino saludable
}

\author{
Resilience at school: a healthy path
}

\author{
Santiago Raúl Campos Aguilar 1,* \\ 1. Ministerio de Educación, Perú
}

\section{RESUMEN}

El objetivo fue valuar el nivel de resiliencia de un grupo de alumnos del $5^{\circ}$ grado de Educación secundaria de la región Cusco, hacer una descripción general de los factores de resiliencia y plantear una propuesta para desarrollar la resiliencia en los colegios evaluados. Se utilizó el diseño descriptivo, basado en una muestra no probabilística con un tamaño muestral de 57 alumnos de dos centros educativos del ámbito urbano y rural de la región Cusco. Se aplicó la Escala de Resiliencia SV-RES de Saavedra y Villalta. Entre los resultados se tiene que el nivel de resiliencia de los alumnos es significativamente bajo; los factores protectores internos como: generatividad, aprendizaje, autoeficacia e identidad obtienen una mayor puntuación y los de menor puntaje hacen referencia a factores protectores externos como: afectividad, vínculos, satisfacción y redes. En conclusión, el nivel de resiliencia de los alumnos del 5 ㅇ grado de educación secundaria de la región del Cusco, es significativamente bajo, cuentan con factores de protección internos tales como: Generatividad, aprendizaje, autoeficacia, identidad y pragmatismo. Esto se debería a que se encuentran en la etapa de la vida de mayor proyección a futuro. Por otro lado, se aprecia no cuentan con factores de protección externos como: afectividad, vínculos, satisfacción y redes. Esta situación puede deberse a que muchos de estos alumnos no cuentan con el respaldo familiar y/o recursos económicos para poder concretar su visión de futuro.

Palabras claves: Riesgo, adversidad, resiliencia, psicología positiva, prácticas de enseñanza, cultura escolar.

\section{ABSTRACT}

The objective was to evaluate the level of resilience of a group of students from 5th grade of secondary education in the Cusco region, make a general description of the factors of resiliency and consider a proposal to develop resilience in evaluated schools. Descriptive, based on a sample design was used not probabilistic with a sample size of 57 students from two schools in the urban and rural area of the Cusco region. Applied the scale of resilience SVRES of Villalta and Saavedra. Among the results is that the level of resilience of students is significantly low; internal protective factors such as: generativity, learning, self-efficacy and identity get a higher score and the score refer to external protective factors such as: affectivity, links, satisfaction and networks. In conclusion, the level of resilience of the students of the 5th grade of secondary education in the region of Cusco, it is significantly low, they have internal protective factors such as: Generativity, learning, self-efficacy, identity and pragmatism. This would be due to that you are in the stage of life of most projection in the future. On the other hand, there is no feature external protective factors such as: affectivity, links, satisfaction and networks. This situation may be that many of these students do not have the family support or financial resources in order to realize its vision for the future.

Keywords: Risk, adversity, resilience, positive psychology, teaching practices, school culture.

Historial del artículo:

Recibido, 06 de noviembre de 2017; aceptado, 14 de diciembre de 2017; disponible en línea, 01 de febrero de 2018

* Máster en Intervención Educativa y Psicológica - Profesor de Ciencias Sociales - Minedu

Correo: santiago.campos@pronabec.edu.pe 


\section{INTRODUCCIÓN}

La vida de los seres humanos está compuesta por un sin número de experiencias positivas; éxito, satisfacción, triunfo y la felicidad, entre otras. Estas experiencias junto a los atributos personales y apoyo recibido sirven de estímulo y sostén para enfrentar y transformar positivamente el rumbo de una vida. Por el contrario, hay muchas personas que han pasado y pasan por experiencias negativas. Como: la pobreza, el maltrato, la pérdida de seres queridos, enfermedad, adicción, problemas familiares, etc.

El Perú no es ajeno a esta realidad; por ejemplo, la Unicef (2013) en su informe anual señala que las defensorías del Niño y el Adolescente del Perú registraron 5,305 casos de violencia contra los niños, niñas. También indica, que se producen 60 nacimientos cada hora; 17 de cada 1,000 niños no llega a cumplir el primer año de vida; $17.5 \%$ de niños y niñas menores de 5 años padecen de desnutrición crónica.

Asimismo, la Fundación Holandesa Benard Van Leer (2012), institución orientada a la acción social y protección infantil, que opera en Perú señala que: en el año 2008, únicamente el $4 \%$ de los niños menores de 3 años (el $2 \%$ en zonas rurales) tenía acceso a programas de aprendizaje temprano. Se calcula que el $41 \%$ de las mujeres que tienen pareja han experimentado violencia por parte de ésta, y ello es más probable en los hogares con niños pequeños: la investigación de la OMS reveló que del $15 \%$ al $28 \%$ experimentó violencia física durante el embarazo, y UNICEF calcula que el $37 \%$ de las madres con niños menores de 5 años sufre violencia física. Esto implica que 1,85 millones de niños menores de 8 años viven en hogares donde se maltrata a sus madres. Además, cerca de la mitad de la población urbana del Perú $(9,6$ millones de personas) vive con al menos una de las privaciones que conforman la vida en un suburbio: aglomeración, construcción precaria de la vivienda, falta de acceso al agua y a los servicios sanitarios, o no propiedad de la tierra. Además, una de cada cinco personas soporta dos o más de esas privaciones.

Por su parte la Comisión Nacional para el Desarrollo y Vida sin Drogas (2013) señala que la edad promedio de inicio del consumo de drogas legales fue de 13.7 años para el alcohol y 13.6 años de edad para el tabaco. Para las drogas ilegales, la edad promedio de inicio del consumo de los inhalantes fue 13 años, que fue el menor promedio; mientras que el mayor promedio lo tuvo la marihuana, con 14.4 años de edad. Se encontró que la edad mínima de inicio de consumo para todas las drogas ha sido de 10 años de edad, a excepción del consumo de tabaco, en el que se encontró una edad mínima de inicio de hasta 8 años de edad.
Como podemos ver las estadísticas son alarmantes, sin embargo, dentro del grupo de personas, que lamentablemente pasaron y pasan por experiencias negativas como las que acabamos de señalar, hay un grupo de personas que logra superar la adversidad y las predicciones devastadoras; que enfrenta su realidad de forma ejemplar, fortaleciéndose y recuperándose exitosamente, sirviendo de ejemplo y modelo a seguir para muchos.

Cuando se estudia el fenómeno de la resiliencia, se trata de comprender la forma en que los seres humanos pueden sobrevivir y superar las adversidades a pesar de vivir en condiciones de pobreza, violencia familiar, enfermedad mental de los padres o a pesar de las consecuencias de una catástrofe natural, entre otras. Al poner en acción esta capacidad, se promueven los procesos que involucran al individuo y su ambiente social, ayudándolo a superar la adversidad y el riesgo. Esto le permite adaptarse a la sociedad y tener una mejor calidad de vida.

En este estudio centraremos nuestro interés en la resiliencia en la escuela, veremos la resiliencia y su importancia, algunas investigaciones y propuestas para su desarrollo y finalmente, presentaremos un estudio piloto con un grupo de alumnos del 5 o grado de Educación Secundaria de la región sur del Perú.

\section{La resiliencia y su importancia}

Según el CEANIM (2014) el término "resiliencia" es un concepto que pasó de la física al área de la psicología. Se entiende por resiliencia la capacidad que tiene un cuerpo físico de recobrar su forma primitiva, cuando se cesa de ejercer presión sobre él.

Desde los años ochenta, el concepto de resiliencia ha ido evolucionando debido al especial interés por parte de los investigadores en el estudio del desarrollo normal de todos los individuos, a pesar de las situaciones difíciles que puedan a travesar. Luego analizar las diferentes definiciones del término resiliencia de autores como: CEANIM (2014); Grotberg (2006); Manciaux (2005); Vanistendael (2010); Barudy (2011); Ungar (2012); Henderson y Milstein (2007); Cyrulnik, Tomkiewicz, Guénard, Vanistendael y Manciaux (2013), podemos señalar que todos destacan la capacidad que tienen todas las personas para superar la adversidad y proyectarse al futuro. La resiliencia es una capacidad universal que permite a la persona o grupo superar la adversidad y seguir viviendo lo mejor posible, sin negar el pasado. Pero es necesario el desarrollo de cualidades mentales de conducta, adaptación así como, la elección de recursos psicológicos, sociales, culturales y físicos que sustentan el bienestar. 


\section{Importancia de la resiliencia}

En las últimas décadas la resiliencia ha ganado importancia en parte debido a la Psicología Positiva, que ha aportado un nuevo enfoque a las investigaciones e intervenciones, más centrado en el desarrollo de las capacidades y fortalezas, y en la promoción y desarrollo de factores resilientes, que sirven como una especie de escudo que conserva la estructura interna de la persona, impidiendo que el estrés al que está expuesta deteriore su salud física o mental (GonzálezTorres, 2011).

Autores como Henderson y Milstein (2003); Grotberg (2006); Ungar (2012); Martin, Seligman, Randal, Gillham, Reivich, Linkins, (2009); Manciaux (2005); Kotliarenco, (2012) señalan que la resiliencia es importante porque permite enfocar la atención desde el déficit a las fortalezas. Cambia la perspectiva: en ves vez de estudiar las debilidades, las carencias y los medios de compensarlas, interesa investigar las fuerzas y cómo usarlas. No se detiene a examinar la patología sino más bien la salud y la adaptación adecuada. El objetivo es ayudar a los individuos y grupos no sólo a enfrentar las adversidades, sino también a beneficiarse de tales experiencias.

Asimismo, podemos agregar que ha suscitado gran interés en diferentes ámbitos profesionales incluso en el ámbito castrense de las Fuerzas Armadas. Con el fin de ayudar a los militares y sus familias a manejar más eficazmente los desafíos de la vida militar y muy especialmente después de las operaciones de combate a recuperarse y reintegrarse de una manera saludable y positiva, el Ejército Norteamericano en el 2009 en cooperación con la Universidad de Pensylvania y Martín Seligman, desarrollaron un programa denominado Master Resilience Training, a la fecha hay más de 19 estudios controlados que comprueban su eficacia. (Reivich, J.K., Seligman, M. E. P. y McBride, S. 2011).

Theis (en Manciaux, 2005) señala que la resiliencia nos invita a positivar nuestra mirada al prójimo y a modificar nuestras prácticas, empezando quizás a observar, identificar y usar mejor los recursos, reducir los riesgos y favorecer las competencias de cualquier persona y sin duda los beneficios para la sociedad son mayores.

\section{Ámbitos de desarrollo de la resiliencia}

Los ámbitos cruciales para que los individuos desarrollen la capacidad de sobreponerse a la adversidad, se adapten a las presiones y problemas que enfrentan y adquieran las competencias social, académica y vocacional necesarias para salir adelante en la vida, son la familia, la escuela y la comunidad. A continuación, ampliaremos cada uno de los ámbitos donde se puede desarrollar la capacidad de resiliencia.

\section{Ámbito familiar}

La familia al ser el elemento básico de la sociedad es fundamental en el desarrollo de factores resilientes en sus integrantes. Investigadores de la Universidad Nacional Mayor de San Marcos (UNMSM) Perú, realizan un estudio con 214 adolescentes para determinar la capacidad de resiliencia y los factores asociados en cual concluye que el factor protector más importante es la familia (Matalinares, Arenas, Yaringaño, Sotelo, Sotelo, Díaz, Dioses, Ramos, Mendoza, Medina, Pezua, Muratta, Pareja, y Tipacti, 2011).

Asimismo, Baruch y Stutman (en Grotberg, 2006) señalan que luego de 15 años, de investigaciones y experiencias clínicas en el Instituto de Salud Mental, han llegado a la conclusión de que el desarrollo de la resiliencia en el ámbito familiar tiene dos componentes. El primero se refiere al amor, la devoción, el cuidado que un niño debe recibir de, al menos, una persona en su vida. En hogares disfuncionales donde la figura paterna está gravemente afectada, se puede optar por un padre sustituto (abuelo, tío o vecino) a fin de satisfacer esa necesidad. Grotberg (2006) señala que la importancia de los mentores o adultos significativos distintos de los padres durante el desarrollo del joven adolescente ha quedado demostrado incluso en aquellas familias bien constituidas y de padres competentes. El segundo componente paradóiicamente, es la exposición a pequeñas dosis de adversidad, desafíos adecuados a su edad y capacidad de control, teniendo en consideración dos aspectos:

1. Significan una oportunidad para dominar la ansiedad que caracteriza a cada etapa particular del desarrollo evolutivo.

2. El desafío es lo suficientemente breve y no muy intenso, de manera que el niño no se sentirá abrumado. A pesar de que experimentará ansiedad, nos aseguraremos que podrá dominarlas.

Ehrensaft y Tousignat (en Manciaux, 2005) informan también que los estudios sobre la resiliencia demuestran el papel central de la familia en la protección psicológica del niño ante la adversidad. Los factores que se identifican con más frecuencia en los estudios empíricos y clínicos son la presencia de al menos un progenitor que apoya y es afectuoso, y la cohesión entre los miembros de la familia. Este reconocimiento del papel esencial de los padres, sobre todo de la madre, procede del análisis situaciones de conflictos armados, o de desastres naturales. Una reacción adecuada de los padres en sucesos traumáticos y la ausencia de psicopatologías tras éstos tienen un papel importante en la prevención de síntomas de angustia del niño. Además, muchos investigadores describen el efecto protector de la ausencia de separación familiar. En suma, la situación de un niño ante una situación de 
estrés intenso podría determinarla no sólo fenómenos intrapsíquicos, sino también las fuerzas que haya en el sistema social familiar al que pertenece.

Gómez y Kotliarenco (2010), al referirse a factores de riesgo y vulnerabilidad familiar los autores hacen una distinción entre estos. Los factores de riesgo son variables que aumentan la probabilidad de un resultado disfuncional o patológico en un individuo - grupo. En la investigación sobre resiliencia, el concepto "riesgo significativo" está en relación con tres posibilidades:

1. Exposición cotidiana y crónica a condiciones sociales adversas, como pobreza.

2. Exposición a un evento traumático, como abuso sexual, o una severa adversidad como la guerra o un terremoto.

3. Una combinación de alto nivel de riesgo con exposición a un evento traumático particular.

En cambio el concepto de vulnerabilidad se refiere a variables que incrementan la susceptibilidad a los efectos negativos de los factores de riesgo, por ejemplo, una historia de depresión o baja cohesión familiar. El modelo clásico del daño, entonces, señala que en presencia de un contexto adverso (múltiples factores de riesgo), si la persona o familia muestra determinadas debilidades en su funcionamiento (factores de vulnerabilidad) el impacto dañino de los problemas y crisis aumentará significativamente, pudiendo tornarse crónico de no mediar nuevos procesos de recuperación y fortalecimiento.

Por otro lado, Kalil (2003 citado en Gómez y Kotliarenco, 2010) señala que en la familia existen factores protectores cuya definición apunta a la función de "escudo" que ciertas variables cumplen sobre el funcionamiento familiar para mantenerlo saludable y competente bajo condiciones de estrés: por ejemplo, las celebraciones familiares, el tiempo compartido o las rutinas y tradiciones familiares. Estos factores actúan atenuando o incluso neutralizando el impacto de los factores de riesgo sobre la trayectoria evolutiva familiar. Asimismo, se identifican los procesos de recuperación, que cumplen una función de "catalizador" de nuevos equilibrios tras el impacto desestructurante de la crisis, por ejemplo, la comunicación, integración y apoyo familiar, la búsqueda de recreación y el optimismo familiar.

\section{Ámbito escolar}

Después de la familia la escuela es el lugar más propicio para que los alumnos experimenten las condiciones que promueven el desarrollo de la resiliencia. Existen numerosas investigaciones y pruebas de que las escuelas como instituciones pueden ser poderosas constructoras de resiliencia. Asimismo, la escuela puede ayudar a los individuos a sobreponerse y convertirse en estudiantes, trabajadores y ciudadanos más competentes y exitosos. Al mismo tiempo, la escuela es considerada como un factor protector $y$ para niños provenientes de familias disfuncionales es considerado un refugio. Por lo tanto, el profesorado ejerce un papel relevante, y por ello, debe conocer las implicaciones de la resiliencia. Pero además, los factores resilientes están vinculados al rendimiento académico (cfr. Grotberg, 2006; Aguirre, 2002; Arranz, 2006; Villalta, 2010; Forés, 2008; Kotliarenco, 2010; Benard 1991, 1996, 2003; Henderson y Milstein, 2008 y Villalta, 2010).

A continuación destacamos algunas investigaciones, ideas y sugerencias de algunos de los autores antes mencionados. Villalta (2010) realiza un estudio con 437 alumnos sobre "Factores de resiliencia asociados al rendimiento académico en estudiantes de contextos de alta vulnerabilidad social". Sus resultados indican que el desarrollo de factores de resiliencia está asociado al mejoramiento del rendimiento académico. Asimismo, el nivel de resiliencia influye en el planteamiento de metas y definición del proyecto de vida de los estudiantes.

Bonnie Benard (en Williams, 2003) señala que el desarrollo de la resiliencia está directamente relacionada con la calidad de la enseñanza y es considerada como la más poderosa influencia en el rendimiento académico. Mientras que la enseñanza de calidad puede significar muchas cosas, cuando a los estudiantes, los consumidores finales de la enseñanza de calidad, se les pide que expliquen lo que significa para ellos, son inequívocos en su respuesta: Un profesor que cuida, que acepta y que se niega a dejar que ellos fallen "no hay excusa".

Por otro lado, queremos subrayar que entre los autores e investigadores que más familiarizados están con el desarrollo de la resiliencia en la escuela son Henderson y Milstein. Sus publicaciones sobre la resiliencia y el bienestar, el desarrollo juvenil positivo y el cambio escolar e institucional se utilizan en más de veinticinco países. Éstos señalan que más que por ningún otro medio, la escuela construye resiliencia en los alumnos a través de la creación de un ambiente de relaciones personales afectivas. Estas relaciones requieren docentes que tengan una actitud constructora de resiliencia, es decir que transmitan esperanza y optimismo (cualquiera que sea la problemática o la conducta pasada del alumno). Es una actitud que dice: "Creo que puedes lograrlo; estás en condiciones, más que en riesgo". Además, las relaciones constructoras de resiliencia también se caracterizan por centrarse en los puntos fuertes de los alumnos. Los profesionales del ámbito educativo deben buscar las fortalezas de cada alumno con la misma minuciosidad que suelen emplear para detectar sus problemas y hacérselas 
ver. Esto no implica pasar por alto las conductas inapropiadas o de riesgo. Sólo significa equilibrar las cosas para que el alumno reciba al menos tanta retroalimentación sobre sus puntos fuertes, como acerca de sus problemas. Al respecto, la bibliografía es clara: las fortalezas de un alumno son las que lo harán pasar de su conducta "de riesgo" a la resiliencia (Henderson y Milstein, 2008).

Finalmente, apuntamos la propuesta de Henderson y Milstein para construir la resiliencia en la escuela, que se conoce como Rueda de la Resiliencia. Está constituida por seis factores que trataremos más extensamente en otro capítulo. Estos son:

1. Enriquecer los vínculos.

2. Fijar límites claros y firmes.

3. Enseñar habilidades para la vida.

4. Brindar afecto y apoyo.

5. Establecer y transmitir expectativas elevadas.

6. Brindar oportunidades de participación significativa.

Los autores indican que la aplicación de esta propuesta ha dado resultados positivos en los alumnos, logrando una concepción más positiva de sí mismos, un mayor apego a la escuela, un mayor compromiso con las reglas y mejor puntuaciones en los tests estandarizados, así como significativos descensos en actividades delictivas, consumo de drogas y suspensiones. Se ha constatado que estos factores son cruciales para fortalecer la resiliencia en niños y adultos (Henderson y Milstein, 2008).

\section{Ámbito comunitario y social}

Antes de referirnos a la resiliencia en el ámbito comunitario debemos señalar que existen dos tipos de adversidades colectivas básicas: los desastres naturales y aquellas producto de la acción del hombre o de la sociedad. Por otro lado, queremos destacar que en el enfoque comunitario de la noción de resiliencia está la aportación latinoamericana. Asimismo, la Asociación Americana de Psiquiatría, tras el atentado del 11 de septiembre del 2001 en Estados Unidos, realizó una serie de investigaciones de resiliencia en el ámbito comunitario. En este contexto, se analiza la capacidad colectiva, más que los atributos individuales, de los grupos humanos o sociedades que confrontan la adversidad y que permiten recuperar el bienestar.

Suárez-Ojeda y Autler (en Grotberg, 2006) luego de observar una gran variedad y sucesión de catástrofes concluyen que la capacidad de respuesta, no está dada por las características individuales, sino por las condiciones sociales, las relaciones grupales y éticas de cada sociedad. Además, señalan que la dinámica observada en diferentes comunidades ha permitido reconocer ciertas condiciones claves frecuentemente presentes en los grupos que superan los desastres con mayor rapidez y éxito. Estos pilares fundamentales son:

1. Autoestima colectiva.

2. Identidad cultural.

3. Humor social.

4. Adecuada gestión gubernamental.

5. Espiritualidad.

Las investigaciones, en el ámbito comunitario y social giran en torno a la pobreza y desastres naturales de gran envergadura. En Latinoamérica, la pobreza y sus múltiples formas de privación es un hecho concreto y fácilmente distinguible. Por esto, el estudio de la Resiliencia y sus alcances, cobra gran importancia para nuestro continente. A continuación centraremos nuestra atención en el contexto latinoamericano.

\section{Estudio empírico en el Perú}

\section{Justificación}

El presente estudio piloto se justifica, debido a que el estado Peruano, viene ejecutando un plan estratégico de reforma educativa que se extiende hasta el 2021. Además, estudios recientes sobre psicología positiva y desarrollo del carácter demuestran que la construcción de la resiliencia en los alumnos es la base de una educación de excelencia. Asimismo, el trabajo resulta novedoso pues aborda la problemática desde una perspectiva positiva y no del déficit de la persona. Además, marca un hito en el inicio de mi formación en la investigación empírica.

\section{Objetivo e hipótesis}

Este estudio piloto se planteó con el objetivo de evaluar a un grupo de alumnos del 5o grado de Educación secundaria de dos colegios de la zona sur del Perú (Cusco).

\section{Objetivos específicos:}

1. Hacer una descripción general de los factores de resiliencia de un grupo de alumnos del $5^{\circ}$ grado de Educación secundaria de la región sur del Perú (Cusco).

2. Comparar los Factores personales de resiliencia tanto a nivel urbano como rural.

3. Describir los factores altos y bajos de la resiliencia en los alumnos.

4. Plantear en un futuro, con los datos obtenidos una propuesta para desarrollar la resiliencia en los colegios evaluados.

Hipótesis:

1. Que el grupo de alumnos del 5ㅇ grado de Educación secundaria de la región sur del Perú 
(cusco), presenten niveles bajos de resiliencia.

2. Que el nivel de resiliencia de los alumnos del colegio rural, esté muy por encima de los del colegio urbano.

3. Que los factores que hacen referencia a metas y objetivos sean los más altos, debido a la etapa de vida en la que se encuentran los alumnos.

\section{MATERIAL Y MÉTODOS}

Se realizó un análisis de tipo, empírico descriptivo. La muestra fue de un tamaño de 57 alumnos provenientes de centros educativos del ámbito urbano y rural de la región sur del Perú (Cusco). 27 de ellos pertenecen al colegio rural y 30 al colegio urbano. Del total de alumnos, el $60 \%$ son varones y $40 \%$ son mujeres cuyas edades fluctúan entre 15 y 19 años de edad.

Se utilizó como instrumento de recolección de datos la Escala de Resiliencia SV-RES (Saavedra y Villalta, 2008) constituida por 60 ítems, para analizar 12 factores de resiliencia. La Escala tiene un formato tipo Lickert que se califica con un rango de 5 opciones de respuesta: (1) Muy de acuerdo, (2) De acuerdo, (3) Ni acuerdo ni desacuerdo, (4) En desacuerdo, (5) Muy en desacuerdo. El puntaje para cada factor está comprendido entre 5 y 25 puntos. La escala alcanzaría un puntaje mínimo 60 y máximo 300 puntos. Cuenta con baremo para la población chilena.

Asimismo, la escala SV-RES se define en el cruce de dos ejes conceptuales a) El eje interactivo de Grotberg y b) El eje de la respuesta o acción resiliente del Modelo Emergente de Saavedra. El cruce de ambos ejes define conceptualmente los 12 factores que evalúa la escala SV-RES. Véase la tabla 1.

En la tabla 2 se muestra un ejemplar de la Escala de Resiliencia SV-RES (Saavedra y Villalta, 2008). Los ítems se agrupan en factores y éstos en categorías.

Los factores de resiliencia que mide el instrumento son:
Factor 1: Identidad. (Yo soy- condiciones de base). Hace referencia al autoconcepto y los valores culturales que son relativamente estables en el tiempo, que caracterizan al sujeto.

Factor 2: Autonomía. (Yo soy/yo estoy-visión de sí mismo). Se refiriere a juicios sobre el vínculo que el sujeto establece consigo mismo para definir su aporte particular al entorno sociocultural. Alude al sentimiento de competencia frente a los problemas, la buena imagen de sí mismo y la independencia al actuar.

Factor 3: Satisfacción. (Yo soy/yo estoy-visión del problema). Se refiere a juicios que desvelan un estado de satisfacción personal, sentimientos de logro y autovaloración desde el cual el sujeto aborda una situación problemática.

Factor 4: Pragmatismo. (Yo soy/yo estoy-respuesta resiliente). Se refiere a juicios que desvelan un sentido práctico de interpretar las acciones que realiza. Alude a una orientación positiva hacia la acción.

Factor 5: Vínculos. (Yo tengo-condiciones de base). Juicios que ponen de relieve el valor de la socialización primaria y redes sociales con raíces en la historia personal. Se refiere a las relaciones vinculares cercanas e intensas en la infancia que definen estructuras de apego que orientan sistemas de creencias.

Factor 6: Redes. (Yo tengo-visión de sí mismo). Juicios que se refieren al vínculo afectivo que establece la persona con su entorno social cercano. Alude a las condiciones sociales y familiares que constituyen un apoyo para el sujeto.

Factor 7: Modelos. (Yo tengo-visión del problema). Juicios que se refieren a personas y situaciones que sirven de guía al sujeto para enfrentar sus problemas. Las experiencias anteriores sirven de referente para abordar la resolución de problemas.

Factor 8: Metas. (Yo tengo-respuesta resiliente). Juicios que se refieren al sentido de la acción para abordar una situación problemática.

Tabla 1

Factores de resiliencia escala SV-RES (Saavedra y Villalta, 2008).

\begin{tabular}{lllll}
\hline $\begin{array}{c}\text { Competencias } \\
\text { Interaccionales de } \\
\text { Grothberg (1995) }\end{array}$ & $\begin{array}{l}\text { Condiciones } \\
\text { de base }\end{array}$ & $\begin{array}{l}\text { Visión de sí } \\
\text { mismo }\end{array}$ & $\begin{array}{l}\text { Visión del } \\
\text { problema }\end{array}$ & $\begin{array}{c}\text { Respuesta } \\
\text { resiliente }\end{array}$ \\
\hline Yo soy, yo estoy & F1: & F2: & F3: & F4: \\
& Identidad & Autonomía & Satisfacción & Pragmatismo \\
Yo tengo & F5: & F6: & F7: & F8: \\
& Vínculos & Redes & Modelos & Metas \\
Yo puedo & F9: & F10: & F11: & F12: \\
& Afectividad & Autoeficacia & Aprendizaje & Generatividad \\
\hline
\end{tabular}


Alude a coherencia de objetivos y acciones hacia un fin claro y realista a perseguir. Implica proyección a futuro, no dejar sin terminar lo iniciado. Tiene que ver con comportamientos como trabajar sin distraerse, ser resistente, metódico y planificador.
Factor 9: Afectividad. (Yo puedo-condiciones de base). Juicios que se refieren al autoreconocimiento y valoración positiva de la vida emocional, los cuales favorecen la flexibilidad en los métodos, hábitos y preferencias, en la adaptación a situaciones nuevas con humor y empatía.

Tabla 2

Escala de Resiliencia SV-RES (Saavedra y Villalta, 2008). Los ítems se agrupan en factores y éstos en tres categorías: identidad, vínculos y afectividad.

\begin{tabular}{|c|c|c|c|c|c|c|}
\hline & Yo soy - Yo estoy & $\begin{array}{l}\text { Muy de } \\
\text { acuerdo }\end{array}$ & Desacuerdo & $\begin{array}{l}\text { Ni acuerdo } \\
\text { ni esacuerdo }\end{array}$ & $\begin{array}{c}\text { En } \\
\text { desacuerdo }\end{array}$ & $\begin{array}{l}\text { Muy en } \\
\text { desacuerdo }\end{array}$ \\
\hline & $\begin{array}{l}\text { Una persona con } \\
\text { esperanza. }\end{array}$ & & & & & \\
\hline 2. & $\begin{array}{l}\text { Una persona } \\
\text { con buena } \\
\text { autoestima. }\end{array}$ & & & & & \\
\hline 3. & $\begin{array}{l}\text { Optimista } \\
\text { respecto del } \\
\text { futuro. }\end{array}$ & & & & & \\
\hline 4. & $\begin{array}{l}\text { Seguro de mis } \\
\text { creencias o } \\
\text { principios. }\end{array}$ & & & & & \\
\hline 5. & $\begin{array}{l}\text { Creciendo como } \\
\text { persona. }\end{array}$ & & & & & \\
\hline & Yo tengo... & & & & & \\
\hline 1. & $\begin{array}{l}\text { Relaciones } \\
\text { personales } \\
\text { confiables. }\end{array}$ & & & & & \\
\hline 2. & $\begin{array}{l}\text { Una familia bien } \\
\text { estructurada. }\end{array}$ & & & & & \\
\hline 3. & $\begin{array}{l}\text { Relaciones } \\
\text { afectivas sólidas. }\end{array}$ & & & & & \\
\hline 4. & Fortaleza interior. & & & & & \\
\hline 5. & $\begin{array}{l}\text { Una vida con } \\
\text { sentido. }\end{array}$ & & & & & \\
\hline & Yo puedo... & & & & & \\
\hline 1. & $\begin{array}{l}\text { Hablar de mis } \\
\text { emociones. }\end{array}$ & & & & & \\
\hline 2. & Expresar afecto. & & & & & \\
\hline 3. & $\begin{array}{l}\text { Confiar en las } \\
\text { personas. }\end{array}$ & & & & & \\
\hline 4. & $\begin{array}{l}\text { Superar las } \\
\text { dificultades que se } \\
\text { me presentan en } \\
\text { la vida. }\end{array}$ & & & & & \\
\hline 5. & $\begin{array}{l}\text { Una vida con } \\
\text { sentido. }\end{array}$ & & & & & \\
\hline
\end{tabular}


Factor 10: Autoeficacia. (Yo puedo-visión de sí mismo). Juicio sobre las posibilidades de éxito que la persona reconoce en sí mismo ante una situación problemática. Implica la autopercepción de capacidad para poner límites, poder controlar los impulsos, responsabilizarse por las acciones y manejar el estrés.

Factor 11: Aprendizaje. (Yo puedo-visión del problema). Juicios que valoran la situación problemática como oportunidad de aprendizaje. Implica aprovechar la experiencia vivida, aprender de los errores, evaluar el propio actuar, y corregir la acción.

Factor 12: Generatividad. (Yo puedo-respuesta resiliente). Juicios que se refieren a la capacidad de pedir ayuda a otros para solucionar situaciones problemáticas. Alude a la habilidad de crear respuestas alternativas frente a los problemas, promoviendo la cooperación o solicitando apoyo.

La escala SV-RES (Para jóvenes y adultos) ofrece una posibilidad real de ser utilizada en la investigación e intervención psicoeducativa, focalizando la acción y permitiendo evaluar con mayor precisión su impacto. Proporciona un puntaje global de resiliencia y por áreas. Lo cual permite intervenir puntualmente en las áreas deficitarias y poder apoyarse en los puntos fuertes del sujeto, ya que en su corrección pueden distinguirse puntajes globales de Resiliencia, como también factores específicos. La escala es apropiada para ser aplicable en toda América Latina.

\section{Procedimiento:}

- Se buscó un instrumento de evaluación de la resiliencia que estuviera validado (baremado) en el contexto latinoamericano. Encontramos la Escala de resiliencia SV-RES para jóvenes y adultos, elaborada por los Psicólogos Eugenio Saavedra de Chile y Marco Antonio Villalta de Perú. El instrumento ha sido baremado en Chile y empleado en numerosas investigaciones sobre resiliencia en diferentes países y contextos de Latinoamérica (Saavedra, E. y Villalta, M. 2008).

- Seleccionamos dos colegios públicos de la Región sur del Perú (Cusco), en los primeros días del mes de diciembre 2013 y tomamos contacto con las autoridades educativas. Les informamos que con el fin de contribuir a la mejora de la calidad educativa de los centros, queríamos realizar un estudio piloto sobre el nivel de resiliencia de los alumnos del 5o grado de educación secundaria; para lo cual les enviamos una solicitud por escrito (vía email), comprometiéndonos a futuro realizar una propuesta para desarrollar la resiliencia en ambos colegios.
- Las autoridades educativas aprobaron nuestra solicitud y designaron a dos profesores para realizar la evaluación, el 14 de diciembre del 2013, enviamos el cuestionario y las pautas de evaluación a los profesores encargados y a finales del mismo mes, nos devuelven el cuestionario para su análisis respectivo.

- En los meses de enero y febrero se realizó la tabulación de los datos y para lo cual se utilizó el programa (software) estadístico SPSS Versión 19.0

- Durante los meses de marzo y abril, se realizó el análisis estadístico (estadísticos descriptivos-mediadesviación típica-frecuencias), tanto para el total de la muestra como para cada colegio (urbano y rural).

Finalmente, podemos decir que siguiendo una secuencia cronológica dentro de los plazos establecidos hemos alcanzado el objetivo planteado en este estudio piloto: evaluar el nivel de resiliencia de un grupo de alumnos del $5^{\circ}$ grado de Educación secundaria en la región sur del Perú (Cusco) y con un instrumento de evaluación apropiado para el contexto latinoamericano. A continuación, de este capítulo presentamos los resultados de este estudio.

\section{RESULTADOS}

Para el análisis de los resultados se utilizaron estadísticos descriptivos, de frecuencias, medias y desviación típica.

En la tabla 3 se puede apreciar que el grupo de alumnos evaluados $(n=57)$ del $5 \%$ grado de Educación secundaria de la región sur del Perú (Cusco), obtienen un puntaje cercano a los 245 puntos, ubicándose en el percentil 36, lo que significa que el nivel de resiliencia es significativamente bajo, de acuerdo a la baremación propuesta por Saavedra y Villalta.

Al analizar los resultados por factores, en la figura 1, notamos que los que obtuvieron mayor puntuación hacen referencia a los factores protectores internos, generatividad, aprendizaje, autoeficacia e identidad. Por otro lado, en la figura 2 podemos ver que los factores de menor puntuación hacen referencia a los

Tabla 3

Promedio general del nivel de resiliencia del grupo de alumnos del 5 o grado de Educación secundaria de la región sur del Perú (Cusco).

\begin{tabular}{ccc}
\hline Total & Media & $\begin{array}{c}\text { Desviación } \\
\text { típica }\end{array}$ \\
\hline 57 & 244,92 & 24,42 \\
\hline
\end{tabular}


factores protectores externos, afectividad, vínculos, satisfacción y redes.

Descendiendo a nivel de los ítems, en la tabla 4 se aprecia que el grupo de alumnos evaluado $n=57$ obtiene puntuaciones altas en los ítems que hacen referencia a factores protectores internos en las dimensiones "YO PUEDO" (proyectarme al futuro y tomar decisiones), "YO SOY" (una persona con metas en la vida y con esperanza) y "YO TENGO" (proyectos a futuro).

Por otro lado, los ítems con puntuación baja hacen referencia a factores protectores externos en las dimensiones "YO TENGO" (acceso a servicios sociales públicos, relaciones personales confiables, personas que estimulan mi autonomía e iniciativa y relaciones afectivas sólidas) y "YO PUEDO" (confiar en las personas). Cabe recordar que los puntajes en los ítems de la escala de resiliencia, se distribuían desde 5 "muy de acuerdo" hasta 1 "muy en desacuerdo".

\section{Resultados por tipo de colegio}

\section{Colegio rural}

En la tabla 5 vemos que los $n=27$ alumnos pertenecientes al colegio rural, en la evaluación del nivel de resiliencia obtuvieron un puntaje promedio de 241,40 puntos, esto equivale a un percentil 33 y significa que el nivel resiliencia es significativamente bajo.

En cuanto a los factores de resiliencia. En la figura 3 , podemos ver que los factores que obtuvieron una puntuación mayor, hacen referencia también a los factores protectores internos, aprendizaje, identidad, autoeficacia, generatividad y metas.

Por otro lado, en la figura 4 vemos que los factores de menor puntuación, hacen referencia a los factores protectores externos, satisfacción, pragmatismo, vínculos, redes y afectividad.

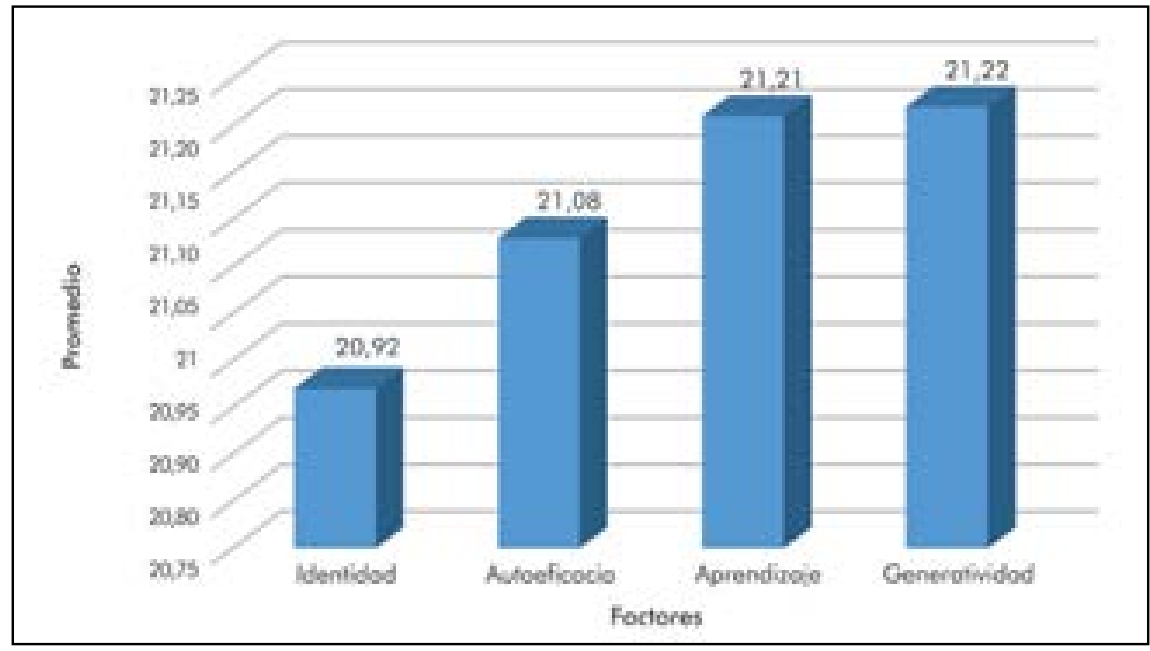

Figura 1. Factores de resiliencia con mayor puntuación.

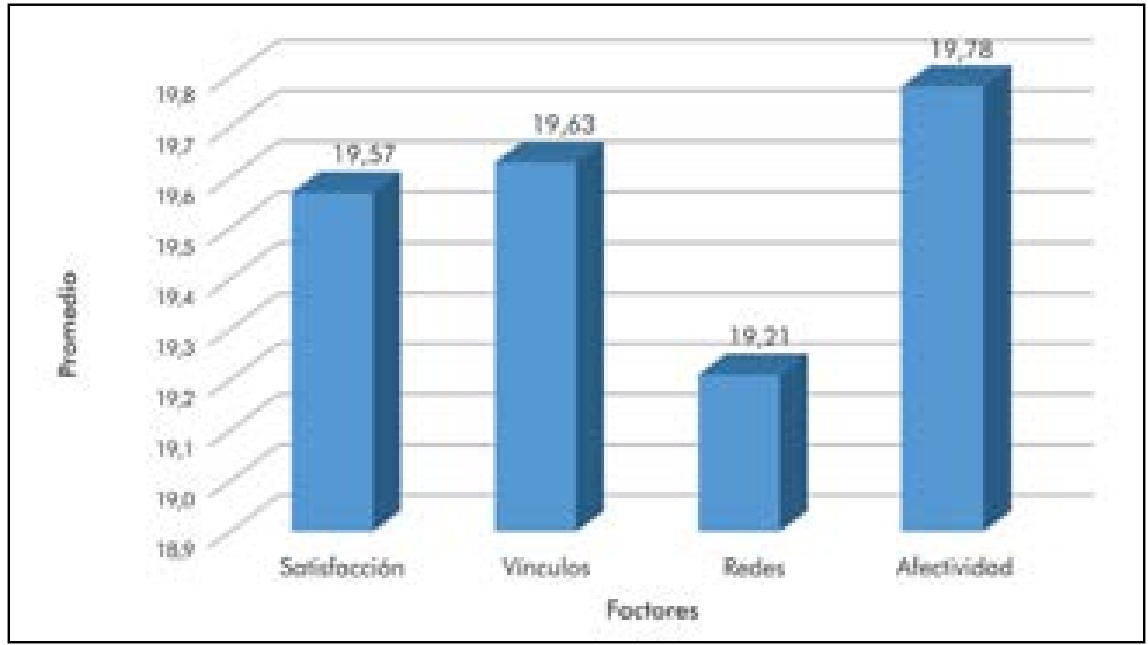

Figura 2. Factores de resiliencia con menor puntuación. 
Tabla 4

Puntaje promedio por ítems (Puntuaciones altas y bajas).

\begin{tabular}{|c|c|}
\hline $\begin{array}{c}n=57: \\
\text { Puntuaciones Altas }\end{array}$ & $\begin{array}{l}\qquad n=57: \\
\text { Puntuaciones Bajas }\end{array}$ \\
\hline $\begin{array}{l}\text { IT: } 60(4,56) \text { YO PUEDO: } \\
\text { Proyectarme al futuro. }\end{array}$ & $\begin{array}{l}\text { IT: } 43(3,47) \text { YO PUEDO: } \\
\text { Confiar en las personas. }\end{array}$ \\
\hline $\begin{array}{l}\text { IT: } 39(4,53) \text { YO TENGO: } \\
\text { Proyectos a futuro. }\end{array}$ & $\begin{array}{l}\text { IT: } 26(3,6) \text { YO TENGO: } \\
\text { Acceso a servicios sociales- } \\
\text { públicos. }\end{array}$ \\
\hline $\begin{array}{l}\text { IT: } 17(4,49) \text { YO SOY: } \\
\text { una persona con metas } \\
\text { en la vida. }\end{array}$ & $\begin{array}{l}\text { IT: } 21(3,65) \text { YO TENGO: } \\
\text { Relaciones personales } \\
\text { confiables. }\end{array}$ \\
\hline $\begin{array}{l}\text { IT: } 1(4,47) \text { YO SOY: } \\
\text { Una persona con } \\
\text { esperanza. }\end{array}$ & $\begin{array}{l}\text { IT: } 29(3,72) \text { YO TENGO: } \\
\text { Personas que estimulan mi } \\
\text { autonomía e iniciativa. }\end{array}$ \\
\hline $\begin{array}{l}\text { IT: } 55(4,42) \text { YO PUEDO: } \\
\text { Tomar decisiones. }\end{array}$ & $\begin{array}{l}\text { IT: } 23(3,72) \text { YO TENGO: } \\
\text { Relaciones afectivas } \\
\text { sólidas. }\end{array}$ \\
\hline
\end{tabular}

Tabla 5

Promedio general del nivel de resiliencia del grupo de alumnos del $5^{\circ}$ grado de Educación secundaria de la región sur del Perú (Cusco).

\begin{tabular}{ccc}
\hline Total & Media & $\begin{array}{c}\text { Desviación } \\
\text { típica }\end{array}$ \\
\hline 27 & 241,40 & 22,14 \\
\hline
\end{tabular}

\section{Colegio urbano}

En la tabla 6 vemos que los alumnos pertenecientes al colegio urbano $(n=30)$, obtienen un puntaje promedio de 248 puntos, correspondiendo a un percentil 36, esto significa que el nivel de resiliencia es significativamente bajo.

En lo referente a factores de resiliencia, en la figura 5 vemos que obtuvieron mayor puntaje también los que
Tabla 6

Promedio general del nivel de resiliencia del grupo de alumnos del 5 grado de Educación secundaria de la región sur del Perú (Cusco).

\begin{tabular}{ccc}
\hline Total & Media & $\begin{array}{c}\text { Desviación } \\
\text { típica }\end{array}$ \\
\hline 30 & 248 & 26,28 \\
\hline
\end{tabular}

hacen referencia a los factores protectores internos, generatividad, pragmatismo, metas, autoeficacia y aprendizaje.

Del mismo modo, en la figura 6 notamos que los factores de menor puntuación, hacen referencia a los factores protectores externos, vínculos, satisfacción y redes.

Finalmente, destacar que el perfil del nivel de resiliencia de los colegios evaluados del contexto urbano y rural es similar, ambos obtienen puntuaciones altas en los factores de protección internos y puntúan bajo en los factores de protección externos. Sin embargo, los factores de protección internos varían, en el colegio rural se destaca el factor identidad, mientras que en los alumnos del colegio urbano el factor pragmatismo.

\section{DISCUSIÓN}

De manera general en el estudio se puede observar que el nivel de resiliencia de los alumnos del $5^{\circ}$ grado de educación secundaria de la región sur del Perú (Cusco) es significativamente bajo. Al comparar los resultados del nivel de resiliencia por tipo de colegio (urbano y rural) se aprecia una ligera diferencia, los alumnos del colegio urbano puntúan tres puntos por encima del colegio rural. Aunque no es una puntuación significativa, este hecho llama la atención, pues en la

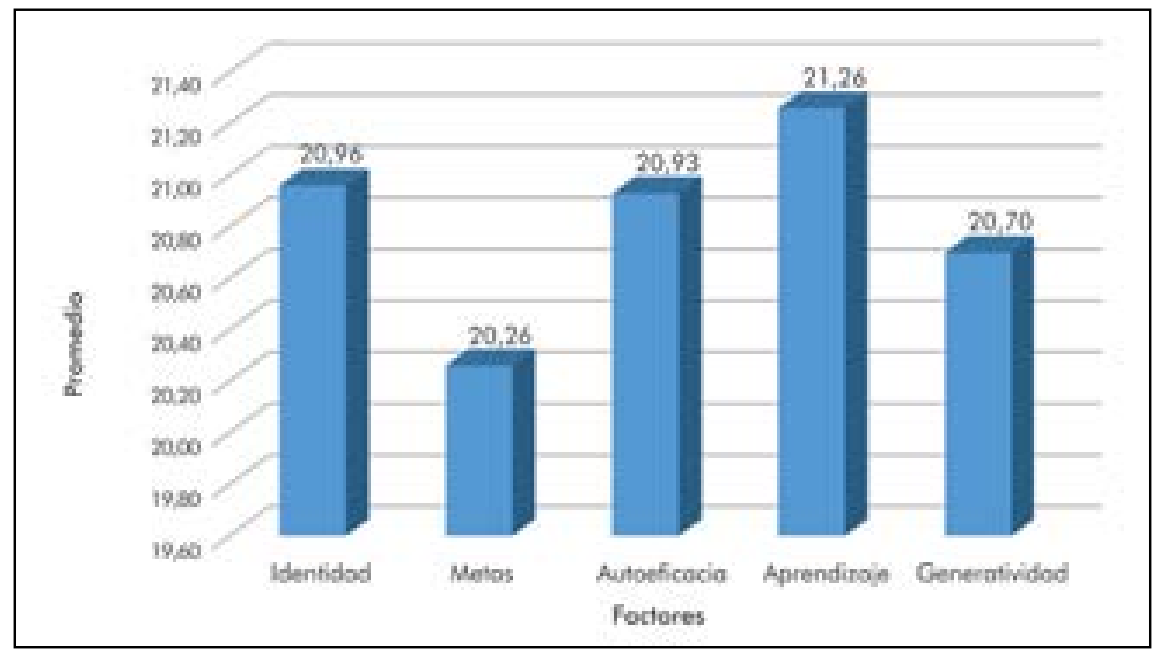

Figura 3. Factores de resiliencia con mayor puntuación $(n=27)$. 


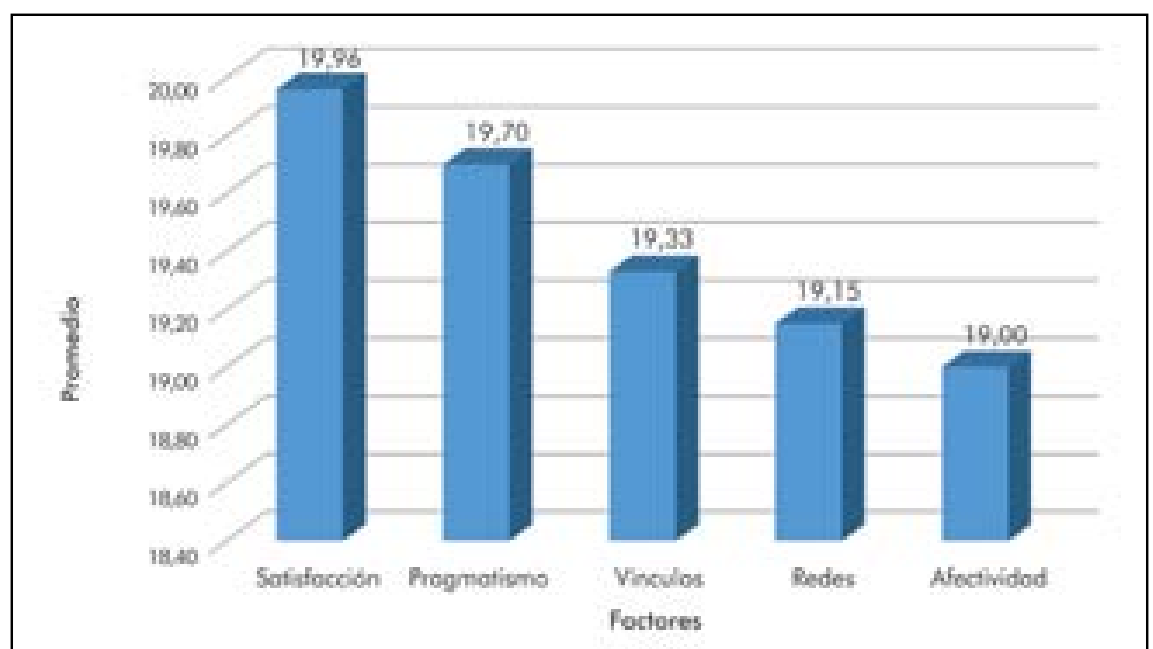

Figura 4. Factores de resiliencia con menor puntuación $(n=27)$.

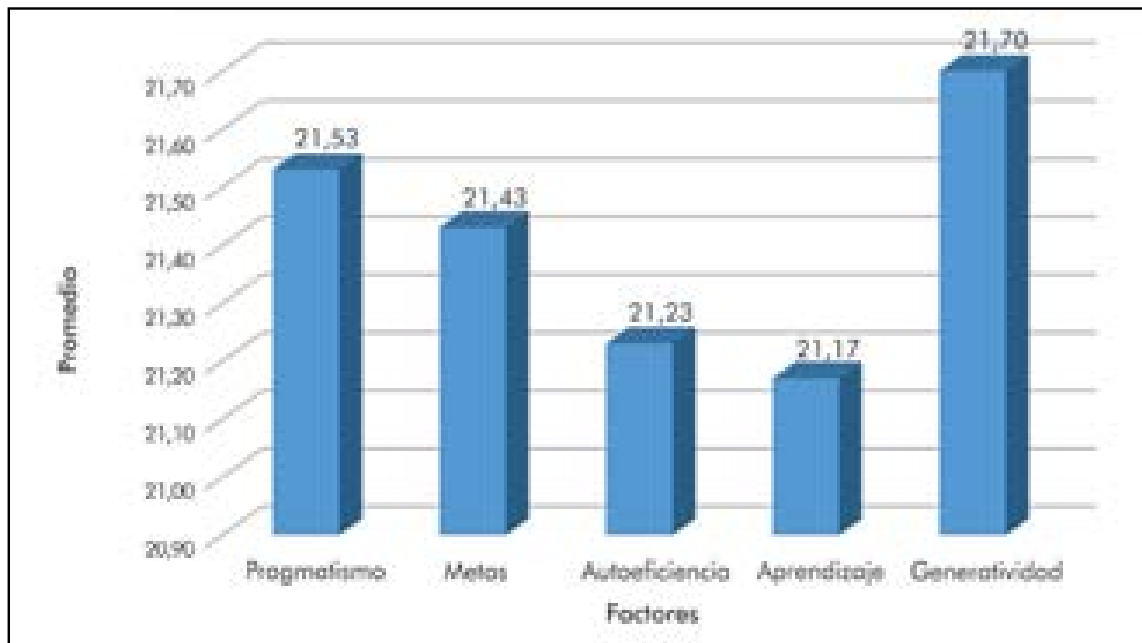

Figura 5. Factores de resiliencia con mayor puntuación $(n=27)$.

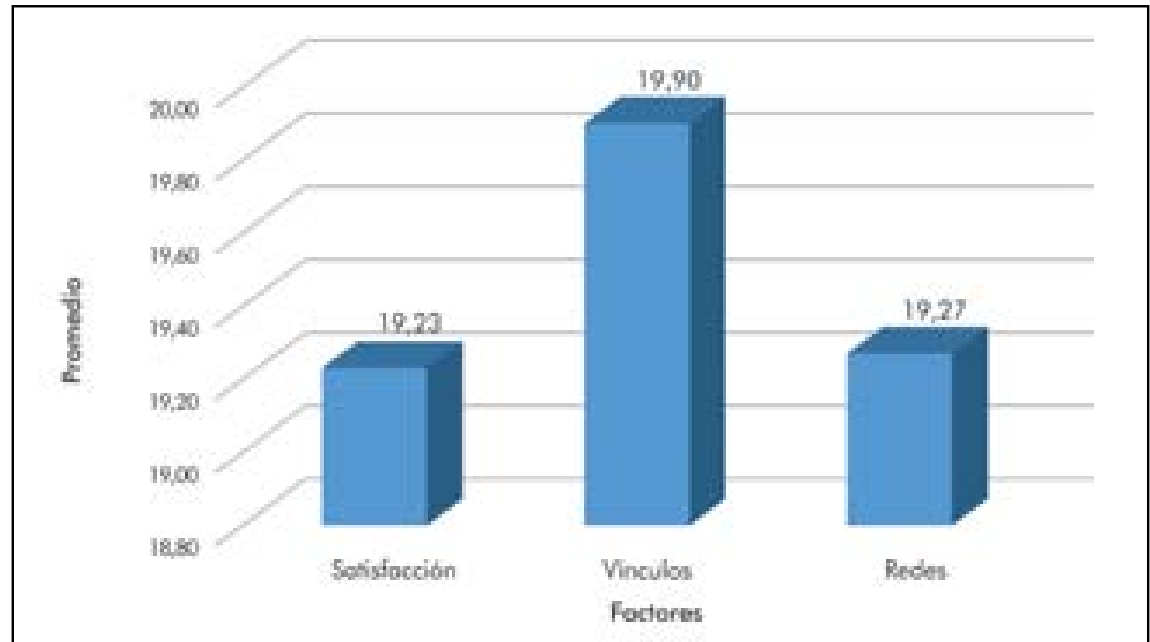

Figura 6. Factores de resiliencia con menor puntuación $(n=30)$. 
bibliografía revisada de Saavedra y Villalta (autores citados) sobre estudios similares, sucede lo contario, los individuos del contexto rural obtienen puntuaciones por encima de los del contexto urbano.

También, se percibe que los 57 alumnos evaluados cuentan con factores de protección internos tales como: Generatividad, aprendizaje, autoeficacia, identidad y pragmatismo. Al respecto, cabe señalar que los alumnos evaluados corresponden al último ciclo de la educación media, etapa de la vida de mayor proyección a futuro. En ese contexto, coincidimos con la explicación que Saavedra hace en un estudio similar donde señala, que las puntuaciones altas en los factores de protección internos se explicarían en cierta manera, por la etapa de la vida en que se encuentran los alumnos, donde se destaca las dimensiones "YO PUEDO" (proyectarme al futuro y tomar decisiones), "YO SOY" (una persona con metas en la vida y con esperanza) y "YO TENGO" (proyectos a futuro).

Por otro lado, se aprecia que los alumnos evaluados no cuentan con factores de protección externos, las puntuaciones bajas están relacionada con las dimensiones YO PUEDO: confiar en las personas; YO TENGO: acceso a servicios sociales públicos, relaciones personales confiables, personas que estimulan mi autonomía e iniciativa y relaciones afectivas sólidas. Asimismo, en los factores de: afectividad, vínculos, satisfacción y redes. Esta situación puede deberse a que muchos de estos alumnos no cuentan con el respaldo familiar y/o recursos económicos para poder concretar su visión de futuro. Por otro lado, los investigadores, dicen que la afectividad y la satisfacción podrían explicarse por el medio social y de consumo actual que enfrentan los jóvenes, donde se avalan y fomentan relaciones afectivas no duraderas, especialmente motivadas por los medios de comunicación. Además, existe un bajo conformismo en diversas áreas, que aumenta la sensación de insatisfacción (cfr. Saavedra, Castro, Saavedra, 2012).

Al diferenciar por colegio, se percibe que tanto los alumnos del colegio rural como del urbano cuentan con factores protectores internos, sin embargo, hay una diferencia en cuanto a la denominación de estos. Los alumnos del colegio rural destacan el factor identidad, mientras que los alumnos del colegio urbano destacan el factor pragmatismo. Esto puede deberse a que los alumnos del colegio rural se sienten mucho más identificados con los valores y conceptos propios de la comunidad. Sin embargo, los alumnos del colegio urbano, tienen menos desarrollado el concepto de vivir en comunidad y los valores compartidos, siendo su actuar mucho más práctico e individualista.

También se percibe que los alumnos de ambos colegios no cuentan con factores protectores externos, pero además, los alumnos del colegio rural se encuentran menos satisfechos que los alumnos del colegio urbano y los alumnos de este último cuentan con menos vínculos. Esto podría deberse a que además, de la etapa de la vida en que se encuentran, los alumnos del colegio rural ven las adversidades de la vida como un reto a superar y no conformarse. En cuanto a los alumnos del colegio urbano que cuentan con menos vínculos, se debería al estilo de vida propio de las ciudades, donde se es más individualista y con pocos vínculos sociales y redes de apoyo.

Este estudio servirá de referencia para en un futuro próximo poder plantear una propuesta para desarrollar la resiliencia en los alumnos de los colegios evaluados. La autopercepción positiva de los alumnos (factores internos), implica un buen punto de partida para generar en ellos respuestas más eficaces frente a sus problemas y desarrollar la resiliencia.

Luego de analizar los hallazgos en la bibliografía revisada para este estudio concluimos en lo siguiente:

- Resiliencia es la capacidad humana para resistir y afrontar el bombardeo incesante de eventos que nos causan sufrimiento, de tal forma que la experiencia nos fortalece, adquirimos mayor confianza en nuestras habilidades y nos volvemos más sensibles a los padecimientos que otras personas estén experimentando, así como más hábiles para generar cambios y minimizar o erradicar las fuentes originarias del estrés o el trauma.

El ambiente es crucial para el desarrollo de la resiliencia, los factores de protección suelen ser el resultado de determinadas condiciones ambientales. Los factores de protección se agrupan en dos categorías: internos y externos. Los factores de protección internos, hacen referencia a elementos positivos propios de nuestro carácter y están relacionados con la competencia social, la habilidad de resolución de problemas, la autonomía y el sentido de propósito y esperanza en el futuro; los factores de protección externos, hacen referencia a las relaciones de apoyo y cuidado, las altas expectativas y oportunidades para la contribución y participación significativa. La atención al buen desarrollo de los factores favorece la resiliencia.

- La resiliencia, es un tema que resulta prometedor porque ha logrado pasar del déficit a las fortalezas, desplaza al modelo médico basado en la patología para aproximarse a un modelo basado en la construcción de fortalezas internas. Se trata de un cambio de paradigma que no atañe sólo al saber médico: en el campo educativo, pensar en términos de resiliencia implica poner el foco en la adquisición y desarrollo de competencias y facultades, en los puntos fuertes y no en el déficit. Algo no tan simple si tenemos en cuenta las situaciones de 
riesgo a las que se hallan expuestos tantos niños y jóvenes: la pobreza extrema, el abandono, el estrés prolongado, los traumas producto de diversas situaciones de violencia, la drogadicción o el alcoholismo de los padres, etc. Los estudios indican, sin embargo, que aquellos niños que han generado un comportamiento resiliente, es decir, que han podido sobreponerse a esas experiencias negativas fortaleciéndose en el proceso, han encontrado alguna persona, ya sea de la familia extensa o de la comunidad, con quien lograron establecer un vínculo positivo. Es aquí donde el rol de la escuela, y en particular el de los docentes, adquiere todo su valor y su poder transformador a pesar de la complejidad. Además, los estudios indican que los estudiantes que aplican estrategias de resiliencia desarrollan una concepción positiva de sí mismos, logran un mayor apego a la escuela y obtienen mejores resultados en las pruebas estandarizadas.

- Los avances e investigaciones sobre resiliencia en diversos ámbitos han permitido elaborar instrumentos de medición y propuestas de intervención en diferentes contextos.

- Finalmente, nuestro interés por el tema reside en el inestimable valor que para muchos niños y jóvenes tiene recuperar su capacidad de superar sus heridas y experiencias de vida negativas. Despertar la convicción de que es posible superar traumas y luchar por una vida digna, devolverles su autoestima, su confianza en el futuro y sus ganas de vivir. Además, convertirse en un ser humano moral que consiga hacer el bien a su alrededor. Éstos son los desafíos por los que apuesta la resiliencia, puede parecer en ocasiones una utopía, como la búsqueda del bien común de la humanidad y de la felicidad. Aunque parezca algo lejano no es imposible.

\section{Agradecimientos:}

A las instituciones, I.E.B.R. Mariscal Ramón Castilla Pucyura - Anta - Cusco, y a la I.E.B.R. Humberto Luna Pacheco - Cusco.

\section{REFERENCIAS BIBLIOGRÁFICAS}

Baruch, R. y Stutman, S. (2006). El yin yang de la resiliencia. En E. H. Grotberg (Ed.), La resiliencia en el mundo de hoy (pp 59-90). Barcelona: Gedisa.

Barudy, J. (2011). Promover el buen trato y los recursos resilientes como bases de la prevención y tratamiento de las consecuencias de la violencia humana. Extraído el 13 de abril del 2014, de http://www.observatorioperu.com/2011/agosto/ Resiliencia.El\%20buen\%20trato.pdf
Benard, B. (1991). Fomento de resiliencia en los niños: Factores de Protección de la Familia, Escuela y Comunidad. Extraído el 09 de mayo de 2014, de http://ecap.crc.illinois.edu/eecearchive/digests/ ed-cite/ed335781.html

Benard, B. (1996). El Fomento de la Elasticidad en los Niños. Extraído el 10 de mayo de 2013, de http:// resilnet.uiuc.edu/library/fomen96s.html

Benard, B. (2003). Turnaround Teachers and Schools. En B. Williams (pp). Closing the Achievement Gap. U.S.A.: Association for Supervision \& Curriculum Development.

BICE. (2014) Definición de resiliencia. Extraído el 05 de Mayo de 2014, de http://www.bice. org/es/actualidades/actualidades-bice/ultimahora/ 1678 -una-definicion-sencilla-de-laresiliencia-por-stefan-vanistendael.html

CEANIM. (2014). Definición de resiliencia. Extraido el 01 de Marzo de 2014, de http://www.resiliencia.cl

DEVIDA. (2013). Encuesta nacional de consumo de drogas. Extraído de http://www.codajic.org/ files/I_ENCUESTA_NACIONAL_CONSUMO_DE DROḠAS_INFRACT̄ORES.pdf

Forés, A. y Grané, J. (2008). La resiliencia: Crecer desde la adversidad. Barcelona: Plataforma.

Fundación Benard Van Leer. (2013). Resiliencia en Programas de Desarrollo Infantil Temprano. Extraido de http://bernardvanleer.org/peru

Grotberg, E. H. (2006). La resiliencia en el mundo de hoy. Barcelona: Gedisa.

Gómez, E. y Kotliarenco, M. A. (2010). Resiliencia Familiar: un enfoque de investigación e intervención con familias multiproblemáticas. Revista de Psicología, Universidad de Chile, 19 (2), 103-131.

González-Torres, M.C. (2011). Hermanos resilientes: taller para afrontar problemas de manera positiva. En O. Lizasoáin, M.C. González-Torres, C. Iriarte, F. Peralta, A. Sobrino, C.E. Onieva, y E. Chocarro (Ed.), Hermanos de personas con discapacidad intelectual: Guía para el análisis de necesidades y propuestas de apoyo (pp 115-164). Logroño (La Rioja): Siníndice.

Henderson, N. y Milstein, M. M. (2008). Resiliencia en la escuela. Buenos Aires: Paídos.

Infante, F. y Lamondl, A. (2006). Resiliencia y biculturalismo: la experiencia de los latinos en Estados Unidos. En E. H. Grotberg (Ed.), La resiliencia en el mundo de hoy (pp 235-270). Barcelona: Gedisa.

Kotliarenco, M. A., Irma, C., Catalina, A. (1996). Resiliencia: Construyendo en la adversidad. Santiago de Chile: CEANIN.

Kotliarenco, M. A. (1997) Estado de arte en resiliencia. Santiago de Chile: CEANIN.

Martin, E. P., Seligman, M., Randal, M. E., Gillham, J., Reivich, K., Linkins, M. (2009). Positive education: positive psychology and classroom interventions. Oxford Review of Education, (35)3, 293-31.

Martínez, P. A. y Liesa, M. (s.f.). La resiliencia en el 
ámbito escolar: alumnos en riesgo de exclusión. Extraído el 01 de abril de 2014, de la Asociación para la Promoción y Desarrollo de la Resiliencia (ADIMA), http://www.addima.org/Documentos/ Articulos/la $\% 20$ resiliencia $\% 20$ en $\% 20$ el\% 20 ambito\%20escolar.pdf

Manciaux, M. (2005). La resiliencia: resistir y rehacerse. Barcelona: Gedisa.

Matalinares, C., Arenas, I., Yaringaño, L., Sotelo, L., Sotelo, L. N., Díaz A., Dioses, A., Ramos, R., Mendoza, P., Medina, Y., Pezua, M., Muratta, R., Pareja, C., Tipacti, R. (2011). Factores personales de resiliencia, y autoconcepto en estudiantes de primaria de Lima Metropolitana. Revista IIPSI: Universidad Nacional Mayor de San Marcos. 14(1), $187-207$.

Melillo, A., Suárez, O. y Nestor, E. (2005). Resiliencia. Descubriendo las propias fortalezas. Buenos Aires: Paidós.

Neil, S. E.(2006). Intensificar la resiliencia en el grupo familiar: un enfoque transgeneracional hacia el cambio positivo en las familias disfuncionales. En E. H. Grotberg (Ed.), La resiliencia en el mundo de hoy (pp 91-130). Barcelona: Gedisa.

Newman, R. (2006). Tras el desastre: la Campaña de Educación Pública de APA contribuye a reconstruir la resiliencia. En E. H. Grotberg (Ed.), La resiliencia en el mundo de hoy (pp 301-342). Barcelona: Gedisa.

Orteu, R. M. (2012). Escuelas resilientes. Extraído el 02 de abril de 2014, de http://www.avntfevntf.com/ imagenes/biblioteca/Trabajo\%203\%C2\%BA\%20 BI\%201 1-12\%20-\%20Orteu,\%20Meritxell.pdf

Reivich, K. J., Seligman, M., McBride, S. (2011) Master resilience training in the U.S. Army. American Psychologist, Vol. 66 (1), 25-34.

Saavedra, E. y Villalta, M. A. (2008). Escala de resiliencia. Santiago Chile: CEANIM.
Saavedra, E., Castro, A., Saavedra, P. (2012). Autopercepción de los jóvenes universitarios y la resiliencia: costrucción de sus particularidades. Extraido el 05 de mayo del 2014 de http://www. ive.edu.co/revistasive/index.php/katharsis/article/ view/174/310.

Salgado Lévano, A. C. (2005). Métodos e instrumentos para medir la resiliencia: una alternativa peruana. Liberabit. Revista de Psicología, 10-11( ) 41-48.

Suárez-Ojeda, E. N. y Autler, L. (2006). La resiliencia en la comunidad: un enfoque social. En E. H. Grotberg (Ed.), La resiliencia en el mundo de hoy (pp 271-299). Barcelona: Gedisa.

Tomkiewicz, S. (2013). El surgimiento del concepto. En B. Cyrulnik, S. Tomkiewicz, T. Guénard, S. Vanistendael, M. Manciaux, (Ed.), El realismo de la esperanza (pp 33-50). Barcelona: Gedisa.

Ehrensaft, E. y Tousignat, M. (2006). Ecología humana y social de la resiliencia. En M. Manciaux (Ed.), La resiliencia: resistir y rehacerse (pp 159-174). Barcelona: Gedisa.

UNICEF. (2013). Todos los niños y las niñas cuentan. Extraído de http://www.unicef.org/peru/spanish/ Informe-Anual-UNICEF-Peru-2013.pdf

Ungar, M. (2012). ¿ What is Resilience? Extraido el 01 de Marzo de 2014, de http://resilienceresearch. org/

Uriarte, J. d. D. (2006). Construir la resiliencia en la escuela. Revista de Psicodidáctica, 11(1) 7-23.

Villalta, M. A. (2010). Factores de resiliencia asociados al rendimiento académico en estudiantes de contextos de alta vulnerabilidad social. Revista de Pedagogía, 31 (88) 159-188.

Villalta, M. A. \& Saavedra, E. (2012). Cultura escolar, prácticas de enseñanza y resiliencia en alumnos y profesores de contextos sociales vulnerables. Universitas Psychologica, 11(1), 67-78. 